\title{
MULHERES NA GESTÃo DE TOPO: A PROBLEMÁTICA DO GAP DE GÊNERO E SALARIAL
}

\section{RESUMO}

Neste estudo pretende identificar-se e verificar-se a presença de um diferencial de género em cargos de gestão de topo, em Portugal. Tenta estudar-se a representatividade das mulheres em cargos de liderança e tenta, também, perceber se a questão da liderança é um problema da passagem de cargos de gestão intermédia para lugares de gestão de topo. Pretende-se, ainda, identificar e verificar a presença de um diferencial de salários entre homens e mulheres que ocupam cargos de gestão de topo em Portugal. Tentando ser inovador no que toca ao estudo da liderança, neste estudo desenvolveu-se um indicador que permitirá medir a capacidade de liderança dos indivíduos em cargos de gestão de topo, para perceber se a fraca representatividade das mulheres encontrada se pode dever a uma capacidade inferior de liderança. Os resultados mostram que mulheres e homens, em cargos de gestão de topo, são similares relativamente à capacidade de liderança.

Palavras-chave: Gênero; Salários; Gestão de Topo; Liderança; Diferencial.

\section{WOMEN IN TOP MANAGEMENT POSITIONS: THE GENDER AND SALARY GAP ISSUE}

\begin{abstract}
This study aims to identify and verify the presence of a gender gap in top management positions. This study try to figure out what the representation of women in positions of leadership, in Portugal, and figure out if the issue of leadership is a problem for passing intermediate management positions to top management positions. It also aims to identify and verify the presence of a wage gap between men and women occupying positions of top management. Trying to be innovate in the study of leadership, it has been developed an indicator that allows the measurement of the leadership competence of individuals in top management positions, to understand if the scarcity of women found in this positions is due to lower leadership skills. The results show that men and women, in top management positions, are similar in what concerns to leadership ability.
\end{abstract}

Keywords: Gender; Pay; Top Management; Leadership; Gap. 


\section{MUJERES EN POSICIONES DE ALTA DIRECCIÓN: LA PROBLEMÁTICA DEL GAP DE GÉNERO Y}

\section{SUELDO}

\section{RESUMEN}

Este estudio pretende identificar y verificar la presencia de una brecha de género en los puestos de alta gerencia. Intenta averiguar la representación de las mujeres en posiciones de liderazgo, en Portugal y averiguar si el tema del liderazgo es un problema para pasar desde puestos intermedios a puestos de alta gerencia. También tiene como objetivo identificar y verificar la presencia de un diferencial de sueldos entre hombres y mujeres que ocupan puestos de alta dirección. Intentando ser innovador cuando se trata del estudio del liderazgo, ha desarrollado un indicador que mide la capacidad de liderazgo de los individuos en posiciones de alta dirección, para entender si la débil representación de las mujeres encontrada puede deberse a una menor capacidad de liderazgo. Los resultados muestran que hombres y mujeres en puestos de alta dirección, son similares en cuanto a habilidades de liderazgo.

Palabras-clave: Género; Sueldo; Alta Dirección; Liderazgo; Gap.

Tânia de Matos Gomes Marques ${ }^{1}$ Célia Melo Ferreira ${ }^{2}$

\footnotetext{
${ }^{1}$ Doutora pela Universidade de Salamanca, Espanha. Professora adjunta na área de gestão/organizacional na Escola Superior de Tecnologia e Gestão (ESTG) do Instituto Politécnico de Leiria - IPL. Portugal. E-mail: taniamarques@ipleiria.pt

${ }^{2}$ Mestre em Gestão de Recursos Humanos e Comportamento Organizacional pelo Instituto Superior Miguel Torga, Coimbra, Portugal. Atualmente trabalha numa instituição bancária portuguesa. Portugal. E-mail: celia.meloferreira@hotmail.com
} 


\section{INTRODUÇÃ̃O}

A representatividade das mulheres em cargo de gestão de topo é escassa e muitas vezes precária (Ryan \& Haslam, 2005). Este fator, associado ao diferencial de salários existente entre homens e mulheres que detêm cargos de liderança (Bowlin \& Renner, 2008), torna a questão do diferencial de género e salarial um assunto presente no mundo empresarial atual, merecendo que a temática seja alvo de mais investigação.

De acordo com Robbins (2008) se o individuo a quem é conferida autoridade formal, possuir capacidade de liderança, então a sua gestão será eficaz e bem-sucedida. Mas a crescente competitividade a nível mundial e o efeito da globalização nas estruturas organizacionais (Black \& Brainerd, 2004) torna a liderança um tema cada vez mais importante já que o papel desempenhado pelos líderes pode influenciar diretamente os seus subordinados (Hoffman, Bynum, Piccolo \& Sutton, 2011).

Um condicionante dos estilos de liderança é a diferença de género entre gestores de topo (Moller \& Gomes, 2010). A diferença de género também é uma barreira para atingir cargos hierárquicos mais elevados (Kyrchmeyer, 2002; Pichler, Smpson \& Stroh, 2008) e é, também, uma das justificações do diferencial de salários verificado entre homens e mulheres (Blau \& Khan, 2006; Duarte, Esperança, Curto, Santos, 2010; Vieira, Cardoso, Portela, 2005). Portanto, a escolha de um CEO para uma organização depende, entre outros fatores, do género, género esse que está associado a estereótipos sexuais discriminatórios, e que podem condicionar a escolha de uma mulher para um cargo de gestão de topo (Loureiro \& Cardoso, 2008).

A relação entre o diferencial de salários e a discriminação de género, já foi estudada em Portugal por Vieira et al. (2005) ou Duarte et al. (2010). Contudo, a investigação realizada sobre Portugal, apesar da sua importância e contributo, não relacionou a capacidade de liderança com o diferencial de género nem diferencial salarial dos gestores de topo.

Assim, este trabalho tem como principais objetivos perceber qual a representatividade das mulheres em cargos de liderança, quando assumem papéis de gestão de topo, em Portugal. Tenta identificar e verificar a presença de um diferencial de género nesses cargos, tendo em conta os sectores de atividade. Também se pretende avaliar, através da construção de um indicador inovador para o efeito - ILIDER, se as capacidades de liderança dos indivíduos podem justificar as diferenças na representatividade em cargos de gestão de topo. Um outro grande objetivo é o de analisar a existência de um diferencial de salários entre os ocupantes desses cargos, por género.

Após a introdução, será feita a revisão da literatura sobre diferencial de género, liderança e diferencial de salários entre homens e mulheres em cargos de gestão de topo. Seguidamente apresentar-se-á a parte empírica, sendo analisada uma amostra de 127 empresas privadas que exercem atividade em Portugal, composta por 467 colaboradores, com dados do ano de 2007, pois 2007 foi o ano imediatamente anterior ao início de uma crise que começou nos Estados Unidos da América e rapidamente se alastrou a todo mundo (Colaco, Myers \& Nitkin, 2010). Por fim, serão apresentadas as principais conclusões bem como as principais limitações e futuras linhas de investigação.

\section{GÊNERO: O DIFERENCIAL EM CARGOS DE GESTÃO DE TOPO}

O género, contextualizado na gestão das organizações, tem recebido cada vez mais atenção em todo mundo, quer por parte da comunidade académica quer por parte da imprensa em geral (Francoeur, Labelle \& Sinclair-Desgagné, 2008). Como nota introdutória deve salientar-se a diferença existente entre sexo e género. Sexo refere-se ao estado biológico de ser-se homem ou mulher e género está mais relacionado com papéis estereotipados e traços de personalidade atribuídos pela sociedade (Weichselbaumer, 2004).

Relativamente a mulheres que ocupam cargos de gestão de topo, isto é, com lugar em Conselhos de Administração, estudos realizados pelo European Women's Professional Network (EWPN) revelam que a discriminação de género em cargos de topo está a diminuir na Europa. Os resultados apresentados em 2010 mostram que o número de mulheres presentes na administração das empresas subiu desde 2004, passando de $8 \%$ a 11,7\%. Relativamente à obtenção de cargos de gestão de topo, de empresas europeias, por parte das mulheres, ainda que se verifique um claro progresso, segundo a EWPN, para um total de 4.875 lugares disponíveis nos Conselhos de Administração das empresas analisadas, só 571 lugares são ocupados por mulheres. Apesar de parecer que a discriminação está a diminuir na Europa, no que concerne aos cargos de gestão de topo ocupados por mulheres e segundo a EWPN, em Portugal verifica-se uma fraca representatividade das mulheres em cargos de gestão de topo, fazendo apenas parte dos Conselhos de Administração das grandes empresas $3,4 \%$ de mulheres.

Analogamente aos estudos oficiais como o da EWPN, estudos científicos também denotam que existe uma baixa representatividade das mulheres em cargos de gestão de topo. Helfat, Harris e Wolfson (2006) ou Oakley (2000) concluem isso mesmo com amostras americanas. Também Bertrand e Hallock (2001) confirmam a existência de um diferencial de género nos cargos de gestão de topo, verificando que as mulheres têm uma representatividade de, apenas, 2,4\% em cargos de gestão de topo nos EUA. Em 2010, Colaco e colegas revelam que $51 \%$ de todos os cargos de gestão nos EUA são ocupados por mulheres, mas 
Mulheres na Gestão de Topo: A Problemática do Gap de Gênero e Salarial

que estas representam apenas $14,8 \%$ dos executivos presentes em Conselhos de Administração das grandes empresas cotadas. Os EUA têm, contudo, a maior percentagem de mulheres com lugares em Conselhos de Administração (quadro 1).

\begin{tabular}{|l|l|c|}
\hline \multicolumn{1}{|c|}{ País (ano) } & \multicolumn{1}{c|}{ Fonte (Ranking de empresas) } & $\%$ \\
\hline Austrália (2003) & Top 200 & 8,4 \\
\hline Canada (2003) & Financial Post 500 & 11,2 \\
\hline Irlanda (2004) & 100 Maiores empresas & 4,8 \\
\hline Itália (1999) & 18 das 25 Maiores Empresas & 0,7 \\
\hline Japão (1998) & 2396 Empresas & 3,0 \\
\hline Noruega (2002) & Todas as Empresas Norueguesas & 7,3 \\
\hline Espanha (2002) & Top 300 & 4,6 \\
\hline África do Sul (2004) & Todas as Empresas Públicas e do Estado & 7,1 \\
\hline Suíça (1999) & 25 Maiores Empresas & 4,0 \\
\hline Reino Unido (2003) & Top 100 & 8,6 \\
\hline Estados Unidos (2003) & 500 Maiores Empresas & 13,6 \\
\hline
\end{tabular}

Quadro 1 - Representação Feminina em Conselhos de Administração, no Mundo Fonte: Adaptado de Adams e Flynn, (2005)

Adams e Flynn (2005) dedicaram a sua investigação ao estudo da representatividade feminina em cargos de gestão de topo, na Europa. Identificam Itália como um dos países com menos mulheres que ocupam lugares em Conselhos de Administração, com apenas $0.7 \%$ de representatividade feminina, em 18 das 25 maiores empresas italianas.

Outros autores como Bowlin e Renner (2008), Pichler et al. (2008) ou Moller e Gomes (2010) também confirmam a disparidade existente entre a representatividade de homens e mulheres em cargos de gestão de topo.

Com base na literatura existente, parece existir um diferencial de género apesar da representatividade feminina em cargos de liderança estar a evoluir positivamente mesmo condicionada às barreiras individuais (experiência profissional e idade) e às barreiras estruturais (tamanho da empresa e setor de atividade) que impedem mais mulheres de atingirem o estatuto de gestoras de topo (Adams \& Flynn, 2005).

\section{Efeito Glass Ceiling}

Segundo Kirchmeyer (2002) as mulheres enfrentam vários obstáculos para atingirem o sucesso. Com o decorrer do tempo, a aspiração a promoção por parte das mulheres decresce, verificando-se a existência do fenómeno teto de vidro (glass ceiling). Este fenómeno de teto de vidro, que impede a progressão na carreira das mulheres que ocupam cargos de gestão intermédia, tem vindo a ser estudado por vários autores (Davidson \& Cooper, 1992; Arulapalam, Booth, Bryan, 2007; Pichler et al., 2008 e Duarte et al., 2010a).
O termo teto de vidro (Davidson \& Cooper, 1992) surge para explicar as barreiras que as mulheres enfrentam para evoluir nas suas carreiras. A metáfora do vidro demonstra a invisibilidade destas barreiras. Pode imaginar-se dois pisos de um edifício, sendo que o segundo piso está destinado aos indivíduos que atingem cargos de diretores executivos nas suas organizações e o piso inferior composto por mulheres que estão retidas em cargos de gestão intermédia. Os dois pisos, divididos por um teto de vidro, permitem às mulheres percecionarem os cargos disponíveis, mas não sendo estes atingíveis pelas mesmas. Para Kirchmeyer (2002) as consequências da desigualdade referente ao efeito teto de vidro prevalecem em cargos hierarquicamente superiores compostos maioritariamente por homens e com a existência de um diferencial de salários.

Riach e Rich (2002) referem a estereotipagem das ocupações como sendo uma componente que interfere diretamente com a decisão de um empregador. Um dos principais fatores que pode influenciar a decisão de nomear uma mulher para um cargo de gestão de topo é o setor de atividade. Também Bertrand e Hallock (2010) defendem que existe uma segregação industrial no que toca aos cargos de gestão de topo, sendo esta uma das justificações para a pouca representatividade das mulheres em cargos de liderança. Bertrand e Hallock (2010) consideram que o setor de atividade influencia a escolha dos gestores e concluem que as mulheres não estão representadas da mesma forma em todos os setores de atividade, tendendo a estar mais presentes nas áreas da saúde, serviços sociais e comércio. Referem, ainda, a fraca representatividade de mulheres em setores de atividade como agricultura, construção e indústrias de 
manufatura pesada. Apontam, ainda, a banca como sendo um setor de atividade com um elevado número de mulheres gestoras intermédias, mas, ao mesmo tempo, como sendo a área com menor percentagem de mulheres executivas de topo, em comparação com outras áreas.

A conclusão de que existe uma segregação por setor de atividade, entre homens e mulheres, em cargos de gestão de topo, é compartilhada por Duarte et al. (2010a). Esses autores confirmam a existência do fenómeno teto de vidro nas atividades com elevada concentração de mulheres, dado que existem mais homens do que mulheres em lugares de gestão de topo. Referem que as mulheres estão mais concentradas nas finanças, nos recursos humanos e em áreas de engenharia, considerados como setores com maior número de mulheres. Em seguimento da ideia que o setor de atividade condiciona a escolha do gestor de topo, também Pichler et al. (2008) confirmam a existência do fenómeno teto de vidro em cargos de gestão de topo, afirmando que as mulheres continuam a ser excluídas destes cargos, em particular em setores de atividade tradicionalmente masculinos (setores industriais e técnicos).

A literatura existente demonstra, portanto, que as mulheres têm menor representatividade do que os homens em cargos de gestão de topo (Kirchmeyer, 2002; Adams \& Flynn, 2005; Colaco et al., 2010) e também que existe uma segregação de género, em cargos de gestão de topo, por setor de atividade (Riach \& Rich, 2002; Pichler et al., 2008; Bertrand \& Hallock, 2010; Duarte et al., 2010a). Assim, para além deste estudo testar a representatividade feminina, ir-se-á, também, testar se o género de encontra uniformemente distribuído pelos diferentes setores de atividade, nos cargos de gestão de topo, colocando as seguintes hipóteses:

H1: Em termos de género, os cargos de gestão de topo apresentam uma proporção desfavorável para as mulheres, quando comparada (a proporção) com o total da população ativa de Portugal.

$\mathbf{H}_{2:}$ Em cargos de gestão de topo, o género não está uniformemente distribuído entre setores de atividade.

\section{Liderança}

Nem todos aqueles que possuem cargos de chefia, possuem capacidades de liderança e muitas vezes nem todos os que são líderes possuem cargos de chefia (Robbins, 2008), pelo que se pode assumir que um gestor de topo não é, necessariamente, um líder. Para liderar, o indivíduo tem que ter a capacidade de mobilizar os outros e influenciá-los a atingirem determinados objetivos (House, 1996; Robbins, 2008). Assume-se que liderar é um dos papéis do gestor (Mintzberg, 1971) e parece ser consensual que o papel do gestor a liderar as equipas é preponderante no sucesso de qualquer organização. A escolha de um líder é fundamental ao ter influência direta no desempenho dos colaboradores e no desempenho da organização (Robbins, 2008; Moller \& Gomes, 2010) pelo que essa escolha (do líder) deve ser ponderada e sem sofrer de estereótipos, na busca do objetivo último das organizações: o melhor desempenho.

Kulich, Trojanowski, Ryan, Haslam e Renneboog (2011) fazem referência à pouca representatividade das mulheres em cargos de liderança, indicando que cargos de gestão de topo estão muitas vezes associados aos traços masculinos e que as mulheres podem ser percebidas como não possuidoras destes traços. Esta pode ser uma das razões para se encontrarem poucas mulheres em Conselhos de Administração das grandes empresas. Kulich et al. (2011) fazem, ainda, referência à performance das empresas onde mulheres exercem cargos de liderança, como sendo condicionante da perceção da capacidade de liderança das mulheres. Segundo Oakley (2000) o estereótipo cultural de líder pertence, tradicionalmente, ao sexo masculino, sendo que as mulheres são percecionadas como lideres menos capazes, já que a liderança feminina é, tendencialmente, uma liderança de partilha de informação e poder, o que funciona bem em cargos de gestão intermédia, mas não em cargos de gestão de topo.

A problemática do "think manager - think male", isto é, pensar em gestão é pensar em masculino (Ryan \& Haslam, 2007, p. 553) parece que ainda se impõe, fazendo com que as mulheres sejam preteridas, em relação aos homens, para cargos de liderança. Nesta linha, os homens são vistos, pelos seus colaboradores e por quem os promove, como mais capazes e tendo as caraterísticas necessárias para uma liderança eficaz. As mulheres estão pouco representadas em cargos de liderança porque não são percecionadas pelos outros como tendo caraterísticas de líderes (Ryan \& Haslam, 2007). Moller e Gomes (2010) fazem um estudo sobre qual o impacto da liderança feminina na implicação organizacional (nível de vinculação do individuo à organização) e, também, constatam que é invulgar encontrar mulheres em altos cargos, em especial cargos de topo relacionados com o poder político ou económico.

Uma vez que Ryan e Haslam (2007), Moller e Gomes (2010) e Kulich et al. (2011) referem a questão da liderança como fundamental para perceber a representatividade das mulheres na gestão de topo, no presente estudo propõe-se avaliar a capacidade de liderança de homens e mulheres através da construção de um indicador a que se deu o nome de ILIDER. O indicador ILIDER - detalhado na secção de metodologia - contempla a experiência do colaborador no cargo, o tamanho da empresa, a performance financeira (ROS) e o crescimento ocorrido em termos de vendas. Assim, coloca-se a seguinte hipótese:

H3: Existe uma diferença significativa na pontuação do indicador ILIDER, entre géneros, em cargos de gestão de topo. 


\section{SALÁRIOS: O DIFERENCIAL EM CARGOS DE GESTÃO DE TOPO}

A diferença de género entre gestores de topo é referida por alguns autores, como sendo condicionante e, também, como uma das justificações do diferencial de salários verificado entre homens e mulheres (Blau \& Khan, 2006; Duarte et al., 2010; Vieira, Cardoso \& Portela, 2005). A questão do diferencial de salários afigura-se como sendo transversal a todos os setores de atividade, bem como a todos os cargos hierárquicos, sendo que as mulheres ganham em média menos do que os homens em todas as funções (Blau \& Khan, 2000).

$\mathrm{Na}$ análise ao censos americano do ano 2000, Miller (2009) constata que existe um diferencial de salários entre homens e mulheres nos EUA, independentemente do setor, concluindo que as mulheres recebem menos, à hora, do que os homens. Riach e Rich (2002) encontraram formas de tratamento diferentes em relação às mulheres (com menos cortesia) e também ofertas de salários mais baixos relativamente aos homens. Pichler et al. (2008) afirmam que as mulheres continuam a ter pouca expressividade numérica em cargos de gestão de topo e concluem que existe um diferencial de salários quando atingem cargos de topo, sendo que as mulheres têm rendimentos significativamente mais baixos do que os homens.

Dados demonstram que, mundialmente, as mulheres ganham menos que os homens devido a, por exemplo, existência de diferenciação de salários na mesma ocupação, diferenciação de salários para funções idênticas ou diferenças entre os empregos desejados e os empregos disponíveis (Anker, 1997).

Com base na literatura existente parece verificar-se, portanto, a existência de um diferencial de salários entre homens e mulheres, diferencial esse que se mostra desfavorável às mulheres.

\section{Diferenças entre retribuição base e retribuição variável em cargos de gestão de topo}

O diferencial de salários, entre homens e mulheres em cargos de gestão de topo, é um tema amplamente discutido entre vários autores como Pichler et al. (2008), Bowlin e Renner (2008), MunozBullón (2010) ou Kulich et al. (2011). Arulapalam et al. (2007) analisam uma base de dados composta por 11 países Europeus, desde o ano de 1995 até 2001, e fazem um estudo, por setor, da distribuição de salários desses países. Concluem que o diferencial salarial aumenta à medida de que se sobe na distribuição salarial, ou seja, nos cargos hierárquicos mais elevados.

Aprofundando um pouco o tema, Bowlin e Renner (2008) concluem que nas 500 pequenas e médias empresas da Standard and Poor's, os salários totais anuais das mulheres executivas são consideravelmente mais baixos do que o dos homens, sendo que as mulheres estão em desvantagem. Contudo, esta diferença acentua-se quando medida a componente variável do salário. As diferenças salariais não só ao nível do salário total. O diferencial de salários aumenta substancialmente quando contabilizada a retribuição variável (Munoz-Bullón, 2010; Kulich et al., 2011). Apesar da aplicação de um sistema de remuneração variável enfrentar inúmeros desafios (Vasconcelos, Silva, Neto \& Theotônio, 2013), afigura-se um diferencial de salários, por género, nesta componente do salário e que merece, portanto, ser investigada com maior detalhe.

Mohan e Ruggiero (2007) estudam os salários dos executivos de topo americanos em relação ao ano de 2002 e comparam a performance financeira da empresa, a retribuição total e a influência do género do CEO. Esses autores concluem que as mulheres obtêm menor compensação quando medida a componente variável do salário. Munoz-Bullón (2010) exalta as diferenças entre o salário base e variável, em que as mulheres que atingem posições de gestão de topo recebem menos $7 \%$ na sua compensação total relativamente aos homens. Munoz-Bullón (2010) expõe que a componente do salário base não explica o diferencial de salários entre homens e mulheres em cargos de gestão de topo, mas que o salário variável será a mais importante causa para a existência deste diferencial. Também no Reino Unido, Kulich et al. (2011) identificam um diferencial de salários, entre homens e mulheres, quer ao nível da remuneração base bem como da remuneração variável, em executivos que ocupam lugares em Conselhos de Administração de empresas cotadas. Esses autores referem, ainda, que as mulheres constituintes do seu estudo ganham menos $19 \%$ do que os homens que desempenham funções similares, verificando que os salários base são mais baixos mas, no caso da retribuição variável, o diferencial é colossal, podendo ser quatro vezes mais elevado no caso dos homens.

Após revisão da literatura relativamente à retribuição base e variável dos gestores de topo, no presente estudo ir-se-á testar se a retribuição anual total (retribuição anual total inclui remuneração base e remuneração variável) e retribuição variável são significativamente diferentes entre géneros, com as seguintes hipóteses:

H4: O salário total é significativamente diferente entre géneros, nos cargos de gestão de topo.

H5: O salário varável é significativamente diferente entre géneros, em cargos de gestão de topo. 


\section{METODOLOGIA}

\section{Objetivo do estudo}

Com base na literatura revista, a representatividade feminina em cargos de gestão de topo mantém-se pouco expressiva e, neste estudo, analisar-se-á o panorama português. Para esse efeito será efetuado um teste para averiguar se a distribuição de homens e mulheres na população ativa, relativamente ao ano de 2007, se encontra em linha com a distribuição de homens e mulheres em cargos de gestão de topo. Ir-se-á, também, averiguar se o género se encontra igualmente distribuído pelos diferentes setores de atividade.

Como a fraca representatividade feminina poderá estar relacionada com a perceção de indicadores de capacidade de liderança que as empresas têm relativamente a homens e mulheres na respetiva nomeação para um cargo de gestão de topo, ir-se-á construir e analisar um indicador para o efeito ILIDER. Neste estudo pretende dar-se um contributo inovador relativamente à investigação em liderança, propondo-se a desenvolver um indicador que permita a quantificação de características de liderança composto pelas variáveis: tamanho, crescimento das vendas, rentabilidade da empresa e experiência no cargo. Com este indicador, espera ser-se que seja possível proporcionar uma explicação empírica para a pouca representatividade feminina nestes cargos, tentando perceber se existem diferenças estatisticamente significativas entre homens e mulheres relativamente à liderança. Para atestar quanto à relevância do indicador ILIDER, este indicador foi desenvolvido considerando a quantificação de características de liderança que se esperam refletidas a dois níveis: na organização (através do ROS) e nos indivíduos (através dos salários).

Para a realização deste estudo foi utilizada uma amostra de 127 empresas privadas que exercem atividade em Portugal, composta por 467 colaboradores, com dados do ano de 2007. A escolha do ano de 2007 justifica-se por ter sido o ano imediatamente anterior ao ano de partida para uma crise que começou nos Estados Unidos da América e rapidamente se alastrou a todo mundo (Colaco et al., 2010).

Através do SPSS, utilizou-se o teste de Kolmogorov-Smirnov e verificou-se ausência de normalidade. Utilizou-se o teste não paramétrico de Mann-Whitney para comparar médias entre grupos de homens e mulheres, e o teste do Qui-quadrado para verificar se existe associação entre diferentes subgrupos da população. Foi utilizado o teste de correlação Spearman's Rho para verificar se existem correlações entre variáveis, alternativas não paramétricas ao teste de Correlação de Pearson (Pestana \& Gageiro, 2008).

\section{Amostra}

A amostra deste estudo é constituída por 440 homens (94\%) e 27 mulheres (6\%), com idades compreendidas entre os 32 e os 82 anos, sendo que a média de idades se situa nos 48,3 anos, conforme quadro 2. A média de idades dos homens (48,1 anos) é inferior à média de idades das mulheres (50,9 anos) e os homens possuem, em média, mais anos de experiência no cargo (14,3 anos) do que as mulheres (11,9 anos). Relativamente ao tamanho da organização, pode verificar-se que os homens exercem funções em empresas com maior número de colaboradores, em média com 2.145 colabores e no caso das mulheres, em média com 1.229 colaboradores. Os resultados mostram, também, que as empresas com homens em cargos de gestão de topo têm, em média, maior crescimento (10\%), do que as empresas com mulheres em cargos de gestão de topo, que crescem, em média, $5 \%$. Relativamente à performance financeira das empresas (ROS), conforme o quadro 2, empresas geridas por homens revelam uma performance financeira positiva média de $7 \%$, e no caso das mulheres, as empresas demonstram resultados similares, $7 \%$. 
Mulheres na Gestão de Topo: A Problemática do Gap de Gênero e Salarial

\begin{tabular}{|c|c|c|c|c|c|c|}
\hline \multicolumn{2}{|c|}{ Sexo } & Idade & $\begin{array}{c}\text { Experiencia no } \\
\text { cargo }\end{array}$ & Tamanho firma & $\begin{array}{c}\text { Crescimento } \\
\text { firma }\end{array}$ & ROS \\
\hline \multirow{4}{*}{ Homens } & Média & 48,09 & 14,28 & 2144,62 &, 10 &, 07 \\
\cline { 2 - 7 } & $\mathrm{N}$ & 387 & 383 & 440 & 440 & 440 \\
\cline { 2 - 7 } & Desvio Padrão & 7,071 & 8,96 & 3231,65 &, 29 &, 049 \\
\cline { 2 - 7 } & Mínimo & 32 & 1 & 7 &,- 99 &,- 10 \\
\cline { 2 - 7 } & Máximo & 82 & 62 & 14570 & 4,19 &, 20 \\
\cline { 2 - 7 } & \% Total & $93,7 \%$ & $93,9 \%$ & $94,2 \%$ & $94,2 \%$ & $94,2 \%$ \\
\hline \multirow{5}{*}{ Mulheres } & Média & 50,86 & 11,90 & 1229,33 &, 05 &, 07 \\
\cline { 2 - 7 } & N & 26 & 25 & 27 & 27 & 27 \\
\cline { 2 - 7 } & Desvio Padrão & 9,86 & 8,39 & 2087,49 &, 098 &, 04 \\
\cline { 2 - 7 } & Mínimo & 34 & 1 & 60 &,- 11 &,- 02 \\
\cline { 2 - 7 } & Máximo & 67 & 34 & 8322 &, 24 &, 12 \\
\cline { 2 - 7 } & \% N Total & $6,3 \%$ & $6,1 \%$ & $5,8 \%$ & $5,8 \%$ & $5,8 \%$ \\
\hline & Média & 48,27 & 14,14 & 2091,70 &, 10 &, 06 \\
\cline { 2 - 7 } & N & 413 & 408 & 467 & 467 & 467 \\
\cline { 2 - 7 } & Desvio Padrão & 7,29 & 8,94 & 3182,34 &, 28 &, 05 \\
\cline { 2 - 7 } & Mínimo & 32 & 1 & 7 &,- 99 &,- 10 \\
\cline { 2 - 7 } & Máximo & 82 & 62 & 14570 & 4,2 &, 20 \\
\cline { 2 - 7 } & \% N Total & $100,0 \%$ & $100,0 \%$ & $100,0 \%$ & $100,0 \%$ & $100,0 \%$ \\
\hline
\end{tabular}

Quadro 2 - Caraterização de Gestores de Topo, por Gênero

Fonte: Dos autores.

Relativamente à caraterização de homens e mulheres por setores de atividade, podemos analisar no quadro 3 que os homens estão mais representados nos setores dos Serviços (18,9\%), Distribuição (17,7\%) e Indústria e Comércio Eletrónico (16,1\%). As mulheres encontram-se com uma representatividade mais elevada nos setores da Indústria e Comércio Eletrónico $(22,2 \%)$, Bens de Consumo (18,5\%) e Serviços
$(14,8 \%)$. Os homens estão menos representados na Indústria Farmacêutica $(0,2 \%)$ e Industrias Diversificadas (outras indústrias que não eletrónica, química e farmacêutica). A representatividade das mulheres é mais baixa nos setores das Indústrias Diversificadas (3,7\%), Comércio e Componentes Auto; Distribuição; Indústria Química e Serviços Financeiros $(7,4 \%)$.

\begin{tabular}{|c|c|c|c|c|c|}
\hline \multicolumn{2}{|r|}{ Sexo } & Frequência & $\%$ & \% Válida & $\%$ Acumulada \\
\hline \multirow{11}{*}{ Homens } & Bens de consumo & 42 & 9,5 & 9,5 & 9,5 \\
\hline & Comércio e Componentes Auto & 22 & 5,0 & 5,0 & 14,5 \\
\hline & Distribuição & 78 & 17,7 & 17,7 & 32,3 \\
\hline & Indústria e Comércio Eletrónico & 71 & 16,1 & 16,1 & 48,4 \\
\hline & Industria Farmacêutica & 1 & ,2 & ,2 & 48,6 \\
\hline & Industria Química & 28 & 6,4 & 6,4 & 55,0 \\
\hline & Industrias Diversificadas & 20 & 4,5 & 4,5 & 59,5 \\
\hline & Serviços & 83 & 18,9 & 18,9 & 78,4 \\
\hline & Serviços Financeiros & 53 & 12,0 & 12,0 & 90,5 \\
\hline & Telecomunicações & 42 & 9,5 & 9,5 & 100,0 \\
\hline & Total & 440 & 100,0 & 100,0 & \\
\hline \multirow{10}{*}{ Mulheres } & Bens de consumo & 5 & 18,5 & 18,5 & 18,5 \\
\hline & Comércio e Componentes Auto & 2 & 7,4 & 7,4 & 25,9 \\
\hline & Distribuição & 2 & 7,4 & 7,4 & 33,3 \\
\hline & Indústria e Comércio Eletrónico & 6 & 22,2 & 22,2 & 55,6 \\
\hline & Industria Química & 2 & 7,4 & 7,4 & 63,0 \\
\hline & Industrias Diversificadas & 1 & 3,7 & 3,7 & 66,7 \\
\hline & Serviços & 4 & 14,8 & 14,8 & 81,5 \\
\hline & Serviços Financeiros & 2 & 7,4 & 7,4 & 88,9 \\
\hline & Telecomunicações & 3 & 11,1 & 11,1 & 100,0 \\
\hline & Total & 27 & 100,0 & 100,0 & \\
\hline
\end{tabular}

Quadro 3 - Proporção de Gênero por Setor de Atividade

Fonte: Dos autores. 
Mulheres na Gestão de Topo: A Problemática do Gap de Gênero e Salarial

Quanto aos salários verifica-se que, em média, os homens recebem 9.949,74€ de retribuição mensal base, $150.092,31 €$ de retribuição anual total e $37.361,37 €$ no caso da retribuição variável. Quanto às mulheres, recebem em média 9.870,29€ de retribuição mensal base, $145.725,21 €$ de retribuição anual total e $28.773,01 €$ de retribuição variável, conforme quadro 4.

\begin{tabular}{|c|c|c|c|c|}
\hline \multicolumn{2}{|c|}{ Sexo } & $\begin{array}{c}\text { Retribuição Mensal } \\
\text { Base }\end{array}$ & $\begin{array}{c}\text { Retribuição Anual } \\
\text { Total }\end{array}$ & $\begin{array}{c}\text { Retribuição } \\
\text { Variável }\end{array}$ \\
\hline \multirow{4}{*}{ Homens } & Média & 9949,74 & 150092,31 & 37361,37 \\
\cline { 2 - 5 } & $\mathrm{N}$ & 440 & 440 & 331 \\
\cline { 2 - 5 } & Desvio Padrão & 5106,87 & 67752,51 & 43875,26 \\
\cline { 2 - 5 } & Mínimo & 2519,80 & 52670,16 &, 00 \\
\cline { 2 - 5 } & Máximo & 49475,70 & 694123,92 & 404000,00 \\
\hline \multirow{4}{*}{ Tulheres } & Média & 9870,29 & 145725,21 & 28773,01 \\
\cline { 2 - 5 } & N & 27 & 27 & 20 \\
\cline { 2 - 5 } & Desvio Padrão & 2826,50 & 37408,57 &, 00 \\
\cline { 2 - 5 } & Mínimo & 2787,39 & 106840,48 & 67499,04 \\
\cline { 2 - 5 } & Máximo & 15984,00 & 227887,56 & 36872,01 \\
\hline & Média & 9945,14 & 149839,83 & 351 \\
\cline { 2 - 5 } & N & 467 & 467 & 42921,02 \\
\cline { 2 - 5 } & Desvio Padrão & 5001,51 & 66359,28 &, 00 \\
\cline { 2 - 5 } & Mínimo & 2519,80 & 52670,16 & 404000,00 \\
\cline { 2 - 5 } & Máximo & 49475,70 & 694123,92 & \\
\hline
\end{tabular}

Quadro 4 - Caraterização dos Salários Médios, por Gênero

Fonte: Dos autores.

Variáveis

No presente estudo foram definidas as seguintes variáveis: Sexo (Homens/ Mulheres); Idade (em anos); Formação Académica (Com/Sem formação Académica); Experiência no Cargo ou Tenure (em anos); Retribuição Mensal (em euros); Retribuição Anual Total (em euros); Retribuição Variável (em euros); Dimensão da empresa (em número de colaboradores); Crescimento da Firma (em vendas do ano em relação com vendas do ano anterior). A performance financeira foi medida usando a variável Return on Sales (ROS) consistente com outros estudos que mediram a performance com a mesma variável (Bowlin \& Renner 2008; Duarte et al., 2010). Para medir a capacidade de liderança dos colaboradores, foi construído um indicador a que se deu o nome de ILIDER.

Para definir o indicador de liderança ILIDER será necessário medir um conjunto de variáveis que permitam analisar o desempenho do colaborador enquanto líder na função que desempenha. Ryan e Haslam (2007), Moller e Gomes (2010) ou Kulich et al. (2011) estudam a liderança através do uso de questionários, medindo assim a perceção. No presente estudo pretende-se avaliar, de uma forma mais quantitativa e tentando que seja mais objetiva, a capacidade de liderança de homens e mulheres. $\mathrm{O}$ ILIDER é construído através da medição por sistema de pontos das variáveis: experiência do gestor de topo, tamanho da empresa, crescimento da empresa e performance financeira da empresa (ROS).
Blau e Khan (2006) e Duarte et al. (2010) salientam a importância da experiência no cargo (tenure) de gestor de topo como fundamental na decisão de atribuir um cargo de gestão de topo, bem como na diferença salarial. Considera-se, portanto, que a experiência no cargo se torna uma variável indispensável para a mensuração de um indicador de liderança.

A dimensão das empresas é, também, um fator referido por vários autores como sendo influenciador do diferencial de género (Bertrand \& Hallock, 2001; Bowlin \& Renner, 2008; Cardoso \& Winter-Ebmer, 2010; Duarte et al., 2010), em que as mulheres tendem a gerir empresas mais pequenas (com menor numero de trabalhadores e menores retornos financeiros). Assim, no presente estudo, escolheu-se a variável dimensão da empresa como a segunda componente do indicador da capacidade de liderança.

$\mathrm{O}$ crescimento das empresas condiciona o sucesso das mulheres, considerando-se o crescimento das empresas como um fator que condiciona em termos de género em cargos de liderança (Ryan \& Haslam, 2005; Bowlin \& Renner, 2008).

A variável ROS também foi usada para a composição do ILIDER uma vez que o gestor de topo é, tipicamente, avaliado através da performance financeira da empresa onde está inserido através de medidas contabilísticas como por exemplo o ROS, sendo compensado com retribuição variável, quando a empresa obtém uma performance financeira positiva (Bowlin \& Renner, 2008). O ILIDER, pretende, portanto, verificar o nível de liderança que um 
colaborador demonstra e é composto através de um sistema de pontos elaborado da seguinte forma:

1. Experiência no cargo (pontuação de 0 a 10), sendo que a cada 5 anos é atribuido1 ponto.

2. Dimensão empresas (pontuação de 2 a 10), dado existirem 5 grupos, a pontuação atribuída será de 2 em 2 valores.

3. Crescimento (pontuação de 0 a 10 ), sendo que será atribuído 1 ponto por cada $5 \%$ de crescimento, e 0 pontos quando o crescimento é inferior ou igual a zero, para crescimento acima de $45 \%$ a pontuação será 10 .
4. Rentabilidade (pontuação de 0 a 10), sendo que será atribuído 1 ponto por cada $5 \%$ de rentabilidade, 0 pontos quando a rentabilidade é inferior ou igual a zero e para crescimento acima de $45 \%$ a pontuação será 10 .

Somando os pontos - que no máximo totalizam 40 pontos - obtém-se um indicador que se pretende seja potenciador de indicar a capacidade de liderança dos indivíduos, conforme se pode verificar no quadro 5.

\begin{tabular}{|c|c|c|c|c|}
\hline Pontuação & $\begin{array}{c}\text { Dimensão } \\
\left(\mathbf{n}^{\mathbf{0}} \text { colaboradores) }\right.\end{array}$ & $\begin{array}{c}\text { Tenure } \\
(\text { experiência) }\end{array}$ & $\begin{array}{c}\text { Crescimento } \\
(\%) .\end{array}$ & $\begin{array}{c}\text { Rentabilidade } \\
(\mathbf{R O S})\end{array}$ \\
\hline 0 & & 0 & $<0 \%$ & $<0 \%$ \\
\hline 1 & & $1-5$ & $0,1 \%-5 \%$ & $0,1 \%-5 \%$ \\
\hline 2 & $1-9$ & $6-10$ & $6 \%-10 \%$ & $6 \%-10 \%$ \\
\hline 3 & & $11-15$ & $11 \%-15 \%$ & $11 \%-15 \%$ \\
\hline 4 & $10-49$ & $16-20$ & $16 \%-20 \%$ & $16 \%-20 \%$ \\
\hline 5 & & $21-25$ & $21 \%-25 \%$ & $21 \%-25 \%$ \\
\hline 6 & $50-249$ & $26-30$ & $26 \%-30 \%$ & $26 \%-30 \%$ \\
\hline 7 & & $31-35$ & $31 \%-35 \%$ & $31 \%-35 \%$ \\
\hline 8 & $250-500$ & $36-40$ & $36 \%-40 \%$ & $36 \%-40 \%$ \\
\hline 9 & & $41-45$ & $41 \%-45 \%$ & $41 \%-45 \%$ \\
\hline 10 & $>501$ & $>46$ & $>46 \%$ & $>46 \%$ \\
\hline
\end{tabular}

Quadro 5 - Operacionalização da Variável ILIDER

Fonte: Dos autores.

\section{RESULTADOS}

Para comprovar a existência de uma proporção desfavorável às mulheres em cargos de gestão de topo
(H1), comparativamente com a população ativa, foi utilizado o teste do Qui-Quadrado.

Tabela 1 - Proporção da População Ativa e Gestores de Topo, por Gênero

\begin{tabular}{|l|c|c|c|}
\hline & Homens & Mulheres & Total \\
\hline Gestores de Topo & 440 & 27 & 467 \\
\hline População Ativa & 2.986 .300 & 2.632 .200 & 5.618 .500 \\
\hline Total & 2.986 .740 & 2.632 .227 & 5.618 .967 \\
\hline
\end{tabular}

Fonte: Dos autores.

Uma vez que não se dispõe de uma teoria que permita efetuar o cálculo de classes esperadas, decidiu construiuse uma tabela de contingência. 
Mulheres na Gestão de Topo: A Problemática do Gap de Gênero e Salarial

Tabela 2 - Tabela de Valores Esperados para População Ativa e Gestores de Topo (Qui-Quadrados Parciais)

\begin{tabular}{|l|c|c|}
\hline & Homens & Mulheres \\
\hline Gestores de Topo & 440 & 27 \\
\hline População Esperada & 248,23 & 218,77 \\
\hline (o-e)2/e & 148,15 & 168,10 \\
\hline População Ativa & 2.986 .300 & 2.632 .200 \\
\hline População Esperada & 2986491,77 & 2632008,23 \\
\hline (o-e)2/e & 0,01 & 0,01 \\
\hline
\end{tabular}

Fonte: Dos autores.

De acordo com os resultados acima e verificando-se que 316,26 é superior ao valor tabelado de 3,841 , pode afirmar-se que os desvios são significativos, sendo que indivíduos gestores de topo e população ativa têm valores diferentes quanto ao género, confirmando-se assim a primeira hipótese (H1).

Para testar a segunda hipótese (H2) que o género não está uniformemente distribuído entre setores de atividade, em cargos de gestão de topo, foi utilizado, igualmente, o teste do Qui-Quadrado.

Tabela 3 - Gênero por Setor de Atividade (Qui-Quadrado)

\begin{tabular}{|c|c|c|}
\hline \multicolumn{2}{|c|}{ Sexo } & \\
\hline \multirow{3}{*}{ Homens } & $\chi^{2}$ & $151,37^{\mathrm{a}}$ \\
\cline { 2 - 3 } & GL & 9 \\
\cline { 2 - 3 } & Sig. &, 00 \\
\hline \multirow{3}{*}{ Mulheres } & $\chi^{2}$ & $7,33^{\mathrm{b}}$ \\
\cline { 2 - 3 } & GL & 8 \\
\cline { 2 - 3 } & Sig. &, 50 \\
\hline
\end{tabular}

a. 0 células $(, 0 \%)$ com frequência menor que 5. O valor mínimo expetável é 44.0.

b. 9 células $(100,0 \%)$ com frequência expetável menor que 5. O valor mínimo expetável é 3.0.

Fonte: Dos autores.

De acordo com a tabela 3, apenas se confirma a segunda hipótese $(\mathrm{H} 2)$ para o caso das mulheres, em que o valor do teste $(7,33)$ é inferior ao valor tabelado 16,919. Os resultados, portanto, confirmam a segunda hipótese (H2) para as mulheres que refere que em cargos de gestão de topo, o género não está uniformemente distribuídos por setores de atividade.
Relativamente à terceira hipótese (H3), numa primeira fase foi utilizado o teste do coeficiente de correlação Ró de Spearman que mede a intensidade da relação entre variáveis (Pestana \& Gageiro, 2008), para testar se existe relação entre o indicador de liderança (ILIDER) e a retribuição recebida pelos colaboradores.

Tabela 4 - Correlação entre ILIDER e Retribuição Anual Total

\begin{tabular}{|c|c|c|c|c|}
\hline \multicolumn{2}{|c|}{} & ILIDER & $\begin{array}{c}\text { Retribuição anual } \\
\text { total }\end{array}$ \\
\hline \multirow{3}{*}{ Ró de Spearman } & \multirow{3}{*}{ ILIDER } & $\rho$ & 1,00 &,$- 12^{* *}$ \\
\cline { 3 - 5 } & & $\mathrm{Sig}$ & $\cdot$ &, 01 \\
\cline { 3 - 5 } & \multirow{3}{*}{ Retribuição anual total } & $\mathrm{n}$ & 467 & 467 \\
\cline { 3 - 5 } & & $\rho$ &,$- 12^{* *}$ & 1,00 \\
\cline { 3 - 5 } & & $\mathrm{Sig}$ & 461 &. \\
\cline { 3 - 5 } & & $\mathrm{n}$ & 467 \\
\hline
\end{tabular}

$$
* * p_{-} .01
$$

$\rho=$ Spearman's Rho

Fonte: Dos autores. 
As variáveis variam de forma significativa em sentido contrário $(p=-0,12)$, ou seja, as categorias mais elevadas de ILIDER estão associadas às categorias mais baixas de Retribuição Anual total. Os resultados também mostram que apenas cerca de $2 \%$ da retribuição anual é explicada pelo ILIDER.
Numa segunda fase, analisa-se a correlação existente entre o indicador de liderança ILIDER e a retribuição anual total, para homens e mulheres, para verificar se o indicador ILIDER tem uma relação forte com a retribuição anual total.

Tabela 5 - Coeficiente de Relação entre ILIDER e Retribuição Anual Total, por gênero

\begin{tabular}{|c|c|c|c|c|c|}
\hline & \multicolumn{3}{|c|}{ Gênero } & ILIDER & $\begin{array}{c}\text { Retribuição } \\
\text { anual total }\end{array}$ \\
\hline \multirow{12}{*}{ Ró de Spearman } & \multirow{6}{*}{ Homens } & \multirow{3}{*}{ ILIDER } & $\rho$ & 1,00 &,$- 13^{* *}$ \\
\hline & & & Sig &. & ,01 \\
\hline & & & $\mathrm{n}$ & 440 & 440 \\
\hline & & \multirow{3}{*}{ Ret anual total } & $\rho$. &,$- 13^{* *}$ & 1,00 \\
\hline & & & Sig &, 01 &. \\
\hline & & & $\mathrm{n}$ & 440 & 440 \\
\hline & \multirow{6}{*}{ Mulheres } & \multirow{3}{*}{ ILIDER } & $\rho$ & 1,00 &, 12 \\
\hline & & & Sig &. & ,56 \\
\hline & & & $\mathrm{n}$ & 27 & 27 \\
\hline & & \multirow{3}{*}{ Ret anual total } & $\rho$ & ,12 & 1,00 \\
\hline & & & Sig & ,56 & . \\
\hline & & & $\mathrm{n}$ & 27 & 27 \\
\hline
\end{tabular}

$* * \mathrm{p}_{-} .01$

$\rho=$ Spearman's Rho

Fonte: Dos autores.

No caso das mulheres não existe correlação significativa entre ILIDER e retribuição anual total. No caso dos homens verifica-se que existe correlação negativa $(\mathrm{p}=-0,13)$, conforme tabela 5 .

Por fim, utilizou-se o teste de Mann-Whitney para atestar se existem diferenças significativas entre géneros em cargos de gestão de topo, tentando averiguar se a perceção da falta de capacidade de liderança é comprovada através do indicador ILIDER.

Verifica-se que não existem diferenças significativas na distribuição do ILIDER entre géneros ( $\operatorname{sig}=0,598)$. Este facto demonstra que embora existam menos mulheres em cargos de gestão de topo, existindo um gap de género nestes cargos, as mulheres possuem indicadores de liderança idênticos aos homens. Tendo em conta a dimensão da empresa (quanto maior em numero de colaboradores, maior pontuação), o crescimento da empresa (quanto maior crescimento, maior pontuação), a performance financeira (quanto maior percentagem, maior pontuação) e experiência no cargo (quantos mais anos de experiência, maior pontuação), as mulheres possuem idêntico valor para o indicador de liderança. Não se confirma a terceira hipótese (H3), sendo que não existe uma diferença significativa na pontuação do indicador ILIDER, entre géneros em cargos de gestão de topo.

Para atestar quanto à relevância do indicador ILIDER para a quantificação de características de liderança, que se esperam refletidas na organização (através do ROS) e nos indivíduos (através dos salários), foi utilizado o teste do coeficiente de correlação Ró de Spearman entre o indicador ILIDER, ROS e Retribuição Anual Total. 
Mulheres na Gestão de Topo: A Problemática do Gap de Gênero e Salarial

Tabela 6 - Correlação entre ILIDER, ROS e Retribuição Anual Total

\begin{tabular}{|c|c|c|c|c|c|}
\hline & & & ILIDER & ROS & $\begin{array}{r}\text { Retribuição } \\
\text { anual total }\end{array}$ \\
\hline \multirow{9}{*}{ Ró de Spearman } & \multirow{3}{*}{ ILIDER } & $\mathrm{P}$ & 1,00 & ,04 &,$- 12^{* *}$ \\
\hline & & Sig & & ,39 & , 01 \\
\hline & & $\mathrm{N}$ & 467 & 467 & 467 \\
\hline & \multirow{3}{*}{ ROS } & $\mathrm{P}$ & ,04 & 1,00 &, 03 \\
\hline & & Sig & ,385 & &, 47 \\
\hline & & $\mathrm{N}$ & 467 & 467 & 467 \\
\hline & \multirow{3}{*}{$\begin{array}{c}\text { Retribuição anual } \\
\text { total }\end{array}$} & $\mathrm{P}$ &,$- 12^{* *}$ &, 03 & 1,00 \\
\hline & & Sig &, 01 & ,47 & \\
\hline & & $\mathrm{N}$ & 467 & 467 & 467 \\
\hline
\end{tabular}

$* * \mathrm{p}_{-} .01$

$\rho=$ Spearman's Rho

De acordo com a tabela 6, verifica-se que a relação entre ILIDER e ROS é positiva embora não seja uma correlação estatisticamente significativa. No entanto existe uma correlação estatisticamente significativa e negativa entre o indicador ILIDER e a Retribuição anual total.

Para verificar a quarta e quinta hipóteses (H4 e H5), foi realizado o teste de Mann-Whitney, comparando assim a distribuição de salários entre géneros. Para analisar a hipótese que aponta para que o salário total é significativamente diferente entre géneros, nos cargos de gestão de topo (H4), de acordo com tabela 7 , não se rejeita a hipótese nula. Deste modo, embora existam diferenças salariais ao nível da retribuição anual total, entre homens e mulheres em cargos de gestão de topo, as mesmas não são estatisticamente significativas, não confirmando a nossa hipótese (H4). Conforme a tabela 7, também no caso da retribuição variável, não se rejeita a hipótese nula. Deste modo, embora existam diferenças salariais ao nível da componente variável, entre homens e mulheres em cargos de gestão de topo, as mesmas não são estatisticamente significativas, não confirmando a quinta hipótese (H5).

Tabela 7 - Teste Mann-Whitney para Retribuição Anual Total e Variável

\begin{tabular}{|c|c|c|c|c|}
\hline \multicolumn{2}{|c|}{ Salários } & & Homens & Mulheres \\
\hline \multirow{3}{*}{ Ret.Anual Total } & $\mathrm{N}$ & & 377 & 24 \\
\hline & Média & & 200,09 & 215,29 \\
\hline & Sig & ,53 & & \\
\hline \multirow{4}{*}{ Ret Variável } & & & & \\
\hline & $\mathrm{n}$ & & 292 & 18 \\
\hline & Média & & 155,70 & 152,22 \\
\hline & Sig & ,87 & & \\
\hline
\end{tabular}

Fonte: Dos autores.

\section{DISCUSSÃO}

A literatura existente demonstrou que as mulheres têm menor representatividade do que os homens em cargos de gestão de topo (Kirchmeyer, 2002; Adams \& Flynn, 2005; Colaco et al., 2010) e, também, que existe uma segregação de género, em cargos de gestão de topo, por setor de atividade (Riach, Rich, 2002; Pichler et al., 2008; Bertrand \& Hallock, 2010; Duarte et al., 2010a). Assim, testou-se, para o caso português, a representatividade feminina e, também, se testou se o género de encontra uniformemente distribuído pelos diferentes setores de atividade, nos cargos de gestão de topo. Corroborou-se a nossa primeira hipótese (H1), em que se verifica que os cargos de gestão de topo apresentam uma proporção desfavorável para as mulheres.

Também se corrobora a segunda hipótese (H2), isto é, em cargos de gestão de topo, o género não está uniformemente distribuído por setores de atividade. Consequentemente verifica-se a existência do fenómeno do teto de vidro. A teoria do teto de vidro em cargos de gestão de topo é comprovada através dos resultados obtidos, que revelam existirem menos mulheres do que homens em cargos de gestão de topo, ainda que apenas $6 \%$ dos indivíduos da nossa amostra sejam mulheres. Este facto é realçado quando comparamos a população ativa (homens e mulheres) 
com a população de gestores de topo da amostra utilizada (homens e mulheres), em que verificamos que a população esperada de mulheres gestoras de topo é de 219, quando na realidade apenas estão presentes apenas 27 mulheres. Apesar da população ativa feminina ter aumentado em 2007 (e a masculina ter diminuído), as mulheres, neste estudo, continuam numa posição desfavorável quando comparadas com os homens em cargos de gestão de topo. Esta posição desfavorável pode, eventualmente, ser explicada por uma das dimensões definidas por Hofstede (1991), a masculinidade/feminilidade. A masculinidade refere que os papéis sociais de homens e mulheres estão bem definidos (os homens devem ser assertivos, duros e focados no sucesso material e as mulheres devem ser ternas, modestas e preocupadas com a qualidade de vida). Oakley (2000) também defende que a fraca representatividade de mulheres em cargos de liderança de deve a causas culturais e comportamentais, que por competirem entre si, afetam homens e mulheres. Contudo, apenas quando as mulheres forem vistas como indivíduos é que a discriminação no local de trabalho desaparecerá (Loureiro \& Cardoso, 2008).

A problemática do "think manager - think male", isto é, pensar em gestão é pensar em masculino (Ryan \& Haslam, 2007, p. 553) ainda se impõe, fazendo com que as mulheres sejam preteridas, em relação aos homens, para cargos de liderança. De acordo com os estudos de Ryan e Haslam (2007), Moller e Gomes (2010) e Kulich et al. (2011) que referem a liderança como fundamental para perceber a representatividade das mulheres na gestão de topo, o presente estudo propôs-se avaliar a capacidade de liderança de homens e mulheres através da construção de um indicador inovador a que se chamou de ILIDER. $\mathrm{O}$ indicador ILIDER contemplou a experiência do colaborador no cargo, o tamanho da empresa, a performance financeira (ROS) e o crescimento ocorrido em termos de vendas. $\mathrm{Na} \mathrm{H} 3$ pretendeu-se avaliar a existência de uma diferença significativa na pontuação do indicador ILIDER, entre géneros, em cargos de gestão de topo. Após as análises realizadas, não se corroborou a H3 pelo que não existem diferenças significativas na distribuição do ILIDER entre géneros. Este facto demonstra que embora existam menos mulheres em cargos de gestão de topo, existindo um gap de género nestes cargos, as mulheres possuem indicadores de liderança idênticos aos homens. Estes valores mostram que embora as mulheres estejam em minoria, estão presentes em empresas com dimensão igual ou superior às dos homens, obtêm resultados semelhantes no que toca à performance financeira da empresa, bem como no seu crescimento e têm em média os mesmos anos de experiência no cargo. Verifica-se que não existe relação entre o indicador de liderança e a performance financeira da empresa (ROS), mas a relação negativa entre o indicador ILIDER e a Retribuição Anual Total, em particular no caso dos homens, revela uma realidade crua, já que aqueles que apresentam melhores resultados, trabalham em empresas maiores e têm mais experiência, não têm necessariamente melhores salários.

A literatura demonstra que os salários totais anuais das mulheres executivas são consideravelmente mais baixos que o dos homens, sendo que as mulheres estão em desvantagem, mas esta diferença acentua-se quando medida a componente variável do salário (Bowlin \& Renner, 2008) e as mulheres obtêm menor compensação quando medida a componente variável do salário (Mohan \& Ruggiero, 2007). No presente estudo testou-se se a retribuição anual total (inclui remuneração base e remuneração variável) e retribuição variável são significativamente diferentes entre géneros. Em termos de resultados, embora existam diferenças salariais ao nível da retribuição anual total, entre homens e mulheres em cargos de gestão de topo, as mesmas não são estatisticamente significativas, não confirmando a $\mathrm{H} 4$.

Semelhante resultado obteve-se na $\mathrm{H} 5$, embora existam diferenças salariais ao nível da componente variável, entre homens e mulheres em cargos de gestão de topo, as mesmas não são estatisticamente significativas, não confirmando a quinta hipótese (H5). Estes resultados poderão indicar que embora o diferencial de género exista e seja elevado em Portugal, neste caso de 93,864\% desfavorável às mulheres, no que toca aos salários, esta diferença não é estatisticamente significativa, contrariando, assim, outros estudos como o de Pichler et al. (2008), Bowlin e Renner (2008), Munoz-Bullón (2010) ou Kulich et al. (2011).

\section{CONCLUSÃO}

As mulheres, neste estudo, continuam numa posição desfavorável quando comparadas com os homens em cargos de gestão de topo. Esta posição desfavorável pode, eventualmente, ser justificada por causas culturais (Oakley, 2000) e será interessante promover futura investigação nesta matéria.

O presente estudo avaliou a capacidade de liderança de homens e mulheres através da construção de um indicador inovador a que se chamou de ILIDER, contemplando a experiência do colaborador no cargo, o tamanho da empresa, a performance financeira medida em termos de ROS e o crescimento ocorrido em termos de vendas. Após as análises, verificou-se que não existem diferenças significativas na distribuição do ILIDER entre géneros. Concluímos que embora existam menos mulheres em cargos de gestão de topo, existindo um gap de género nestes cargos, as mulheres possuem indicadores de liderança idênticos aos homens.

Em termos de salários, embora existam diferenças salariais ao nível da retribuição anual total, entre homens e mulheres em cargos de gestão de topo, as mesmas não são estatisticamente significativas. 
Semelhante resultado obteve-se na análise das diferenças salariais ao nível da componente variável, entre homens e mulheres em cargos de gestão de topo. Apesar das diferenças salariais da componente variável existirem, as mesmas não são estatisticamente significativas. Estes resultados poderão indicar que embora o diferencial de género exista e seja elevado em Portugal, neste caso de cerca de 93,9\% desfavorável às mulheres, no que toca aos salários, esta diferença não é estatisticamente significativa, contrariando, assim, outros estudos como o de Pichler et al. (2008), Bowlin e Renner (2008), Munoz-Bullón (2010) ou Kulich et al. (2011). Tendo em conta que a maioria da investigação é feita nos Estados Unidos da América (Bowlin \& Renner, 2008; Blau \& Khan, 2007), nos nossos resultados poderão estar refletidas as políticas sociais existentes no espaço europeu, diferentes das políticas americanas (Duarte et al., 2010a).

Mas o que será preciso para que haja igualdade no mercado de trabalho? Tal como Goldin (2014) sugere, deve haver mudanças no mercado de trabalho, especialmente na forma como o trabalho é estruturado e remunerado de forma a aumentar a flexibilidade. Acreditamos que um dos objetivos da sociedade portuguesa, em particular o objetivo das empresas, será estilhaçar o teto de vidro, permitindo assim uma representatividade equitativa entre homens e mulheres na gestão de topo, havendo mais heterogeneidade nos Conselhos de Administração, promovendo assim diferenciação e desempenhos bemsucedidos.

\section{Contribuição}

Este trabalho pretende contribuir para o estado da arte em termos de diferencial de género e salarial a nível da gestão de topo das organizações. Ao desenvolver um indicador de liderança, quantitativo, dá um primeiro passo para uma tentativa de estudo da liderança mais objetiva. Com este trabalho pretende aumentar-se os conhecimentos da temática na Europa, focando-se no caso português. Permite que as organizações e seus gestores possam entender a problemática de forma mais aprofundada e sólida. Contribui no sentido da urgência em estilhaçar o teto de vidro na gestão de topo, em Portugal.

\section{Limitações e Estudos Futuros}

O estudo realizado apresenta algumas limitações que podem, naturalmente, servir como linhas futuras a investigar. No que respeita à validade externa dos resultados obtidos, estes poderão não ser generalizáveis, dado que a amostra recolhida é limitada ao caso de Portugal. Outra limitação reside no facto desta amostra ser apenas de gestores de topo, embora sejam o objeto de estudo, tornando importante analisar o que acontece ao nível da gestão intermédia.
O fato de não se verificar um diferencial ao nível dos salários poderá, eventualmente, estar relacionado com a fraca representatividade das mulheres no presente estudo, apenas $6 \%$, embora os resultados ao nível dos salários se revelem animadores, já que contrariam a tendência de discriminação de gestores de topo ao nível salarial.

Para o futuro, e no sentido de confirmar e generalizar os resultados obtidos, seria desejável aprofundar este estudo e replicá-lo com gestores de topo de empresas pertencentes a outros países além de Portugal. Será interessante averiguar, com outros países, se existem diferenças consoante as diferentes sociedades e suas dimensões culturais. Também seria desejável fazer uma análise longitudinal, tentando analisar a evolução ao longo dos anos, em vez de se limitar apenas a um ano. Dever-se-á controlar o efeito das características individuais no diferencial.

Futuramente é desejável continuar a desenvolver o indicador ILIDER. Já que, na grande maioria, as escalas existentes medem a liderança de forma qualitativa, este indicador poderá ser um bom ponto de partida para estudos futuros medirem a liderança de forma quantitativa.

\section{REFERÊNCIAS}

Adams, S. \& Flynn, P. (2005). Local knowledge advances women's access to corporate boards. Corporate Governance, 13, 6: 836-846.

Anker, R. (1997). Theories of occupational segregation by sex: an overview. International Labour Review, 136, 3: 375-395.

Arulampalam, W., Booth, A. \& Bryan, M. (2007). Is there a glass ceiling over Europe? Exploring the gender pay gap across the wage distribution. Industrial and Labor Relations Review, 60, 2: 163 186.

Bertrand, M. \& Hallock, K. (2001). The gender gap in top corporate jobs. Industrial and Labor Relations Review, 55, 1: 3-21.

Black, S. \& Brainerd, E. (2004). Importing equality? The impact of globalization on gender discrimination. Industrial and Labor Relations Review, 57, 4: 540-559.

Blau, F. \& Kahn, L. (2000). Gender differences in pay. Journal of Economic Perspectives, 14, 4: 75-99.

Blau, F. \& Kahn, L. (2006). The U.S gender pay gap in the 1990s: slowing convergence. Industrial and Labor Relations Review, 60, 1: 45-66. 
Blau, F. \& Kahn, L. (2007). The gender pay gap: have women gone as far as they can?. Academy of Management Perspectives, 1, 1: 7-23.

Bowlin, W. \& Renner, C. (2008). Assessing gender and top-management-team pay in the S\&P mid-cap and small-cap companies using data envelopment analysis. European Journal of Operational Research, 185, 1: 430-437.

Cardoso, A. R. \& Winter-Ebmer, R. (2010). Femaleled firms and gender wage policies. Ilrreview, 64, 1: 141-163.

Colaco, H., Myers, P. \& Nitkin, M. (2010). Pathways to leadership: board independence, diversity and the emerging pipeline in the United States for women directors. International Journal of Disclosure and Governance, 8, 2: 122-147.

Davidson, M. \& Cooper, C. (1992). Shattering the glass ceiling-the woman manager. London: Paul Chapman Publishing.

Duarte, C., Esperança, J. P., Curto, J. D. \& Santos, M. C. (2010). Desmitificação do diferencial de remunerações nas empresas. A segregação como verdadeira determinante. Revista Alcance electrónica, 14, 4: 383-400.

Duarte, C., Santos, M., Curto, J., Duarte, C., Esperança, J. \& Carapeto, M. (2010a). The determinants of gender pay gap in Portuguese private firms. Gender in Management: an International Journal, 25, 6: 438-461.

EWPN Board Women Monitor (2010), acedido em 10/agosto/2011, www.europeanpwn.net

Francoeur, C., Labelle, R. \& Sinclair-Desgagné, B. (2008). Gender diversity in corporate governance and top management. Journal of Business Ethics, 81: 83-95.

Goldin, C. (2014). A grand gender convergence: Its last chapter. American Economic Review, 104, 4: 10911119.

Helfat, C., Harris, D. \& Wolfson, P. (2006). The pipeline to the top: women and men in the top executive ranks of us corporations. Academy of Management Perspectives, 11: 42-64.

Hoffman, B. J., Bynum, B. H., Piccolo, R. F., \& Sutton, A. W. (2011). Person-organization value congruence: how transformational leaders influence work group effectiveness. Academy of Management Journal, 54: 779-796.
Hofstede, G. (1991). Cultures and organizations: software of the mind. Maidenhead, UK: McgrawHill.

House, R. (1996). Path-goal theory of leadership: lessons, legacy, and a reformulated theory. Leadership Quarterly, 7, 3: 323-352.

Kirchmeyer, C. (2002). Gender differences in managerial careers: yesterday, today and tomorrow. Journal of Business Ethics, 37: 5-24.

Kulich, C., Trojanowski, G., Ryan, M. K., Alexander Haslam, S. \& Renneboog, L. D. R. (2011). Who gets the carrot and who gets the stick? Evidence of gender disparities in executive remuneration. Strategic Management Journal, 32: 301-321.

López-Zafra, E., De Amicis, C. \& García-Retamero, R. (2005). El liderazgo transformacional como un proceso grupal: un análisis desde la perspectiva de género. Iberpsicología, 10: 1-10.

Loureiro, P. \& Cardoso, C. C. (2008). O género e os estereótipos na gestão. Revista de Estudos Politécnicos, Vi, 10: 221-238.

Miller, P. (2009). The gender pay gap in the US: does the sector make a difference?. Journal of Labour Research, 30: 52-74.

Mintzberg, H. (1971). Managerial work: analysis from observation. Management Science, 18, 2: B97B110.

Mohan, N. \& Ruggiero, J. (2007). Influence of firm performance and gender on CEO compensation. Applied Economics, 39: 1107-1113.

Moller, M. \& Gomes, J. (2010). Quid vincit? O impacto da liderança feminina na implicação organizacional. Análise Psicológica, 4: 683-697.

Munoz-Bullón, F. (2010). Gender-compensation differences among high-level executives in the United States. Industrial Relations: a Journal of Economy and Society, 49, 3: 346-370.

Oakley, J. D. (2000). Gender-based barriers to senior management positions: understanding the scarcity of female CEO's. Journal of Business Ethics, 27, 4: 321-334.

Pestana, M. H. \& Gageiro, J. N. (2008). Análise de dados para ciências sociais : a complementaridade do SPSS. Lisboa: Edições Sílabo.

Pichler, S., Simpson, P. \& Stroh, L. (2008). The glass ceiling in human resources: exploring the link 
between women's representation in management and the practices of strategic human resource management and employee involvement. Human Resource Management, 47, 3: 463-479.

Riach, P. \& Rich, J. (2002). Field experiments of discrimination in the market place. The Economic Journal, 112: 480-518.

Robins, S. (2008). Comportamento organizacional. São Paulo: Pearson Prentice Hall.

Ryan, M. \& Haslam, S. (2005). The glass cliff: evidence that women are over-represented in precarious leadership positions. British Journal of Management, 16: 81-90.

Ryan, M. \& Haslam, S. (2007). The glass cliff: exploring the dynamics surrounding the appointment of women to precarious leadership positions. Academy of Management Review, L32, 2: 549-572.

Vasconcelos, K., Silva, G., Neto, M. \& Theotônio, K. (2013). O alinhamento da remuneração variável com a estratégia em ambientes de balanced scorecard. Revista Ibero-Americana de Estratégia, 12, 3: 151-182.

Vieira, J., Cardoso, A. \& Portela, M. (2005). Gender segregation and the wage gap in portugal: an analysis at the establishment level. Journal of Economic Inequality, 3: 145-168.

Weichselbaumer, D. (2004). Is it sex or personality? The impact of sex-stereotypes on discrimination in applicant selection. Eastern Economic Journal, 30, 2: $159-186$.

\section{Um especial agradecimento ao Professor Doutor Carlos Duarte $\dagger$}

